\title{
Edge Monophonic Domination Number of Graphs
}

\author{
P. Arul Paul Sudhahar ${ }^{1}$, M. Mohammed Abdul Khayyoom ${ }^{2}$, and A. Sadiquali ${ }^{3}$ \\ ${ }^{1}$ Department of Mathematics, Rani Anna Govt.College(W) \\ Tirunalveli-627 008, Tamilnadu, INDIA. \\ email: arulpaulsudhahar@gmail.com \\ ${ }^{2}$ Department of Higher Secondary Education, \\ Govt.Vocational HSS, Omanoor-673 645, Kerala,INDIA. \\ email: khayyoom.m@gmail.com \\ 3 Department of Mathematical Science, \\ MEA Engineering College,Perinthalmanna-679 325, Kerala, INDIA. \\ email: sadiqmath@gmail.com
}

\begin{abstract}
In this paper the concept of edge monophonic domination number of a graph is introduced.A set of vertices $D$ of a graph $G$ is an edge monophonic domination set (EMD set) if it is both edge monophonic set and adomination set of G. The edge monophonic domination number (EMD number) of $\mathrm{G}, \gamma_{\mathrm{m}_{\mathrm{e}}}(\mathrm{G})$ is the cardinality of a minimum EMD set. EMD number ofsome connected graphs are realized.Connected graphs of order $\mathrm{n}$ with EMD number $\mathrm{n}$ are characterised.It is shown that for any two integers $\mathrm{p}$ and $\mathrm{q}$ such that $2 \leq \mathrm{p} \leq \mathrm{q}$, there exist a connected graph $\mathrm{G}$ with $\gamma_{\mathrm{m}_{\mathrm{e}}}(\mathrm{G})=\mathrm{p}$ and $\gamma_{\mathrm{m}_{\mathrm{e}}}(\mathrm{G})=$ q. Also shows that there is a connected graph $G$ such that $\gamma(G)=p, m_{e}(G)=q$. and $\gamma_{m_{e}}(G)=p+q$.
\end{abstract}

\section{Keywords}

Monophonic number; Edge monophonic number; monophonic domination number;edge monophonic domination number.

\section{AMS Subject Classification: 05C12. \\ 1 INTRODUCTION}

By a graph $G=(V, E)$ we consider a finite undirected graph without loops or multiple edges. The order and size of a graph are denoted by $\mathrm{m}$ and $\mathrm{n}$ respectively. For the basic graph theoretic notations and terminology we refer to Buckley and Harary [1]. For vertices $u$ and $v$ in a connected graph $G$, the distance $d(u, v)$ is the length of a shortest $u-v$ path in $G$. An $u-v$ path of length $d(u, v)$ is called $u-v$ geodesic. A chord of a path $P: u_{1}, u_{2} \ldots u_{n}$ is an edge $u_{i} u_{j}$ with $j \geq i+2$. An $u-v$ path is monophonic path if it is chord less path. A monophonic set of $G$ is a set $M \subset V(G)$ such that every vertex of $G$ is contained in a monophonic path of some pair of vertices of M.The monophonic number of a graph $G$ is explained in [4] and further studied in[2]and [3].

The neighbourhood of a vertex $\mathrm{v}$ is the set $\mathrm{N}(\mathrm{v})$ consisting of all vertices which are adjacent with $\mathrm{v}$. A vertex $\mathrm{v}$ is an extreme vertex if the sub graph induced by its neighbourhood is complete. A vertex $\mathrm{v}$ in a connected graph $\mathrm{G}$ is $\mathrm{a}$ cut vertex of $G$, if $G-v$ is disconnected. A vertex $v$ in a connected graph $G$ is said to be semi - extreme vertex of $G$ if $\nabla(\langle N(v)\rangle)=|N(v)-1|$.A graph G is said to be semi - extreme graph if every vertex of $\mathrm{G}$ is a semi extreme vertex. Every extreme vertex is a semiextreme vertex. Converse need not be true (see remark 2.2 in[2]).An acyclic connected graph is called tree [1].

A dominating set in a graph $G$ is a subset of vertices of $G$ such that every vertex outside the subset has a neighbour in it. The size of a minimum dominating set in a graph $G$ is called the domination number of $G$ and is denoted $\gamma(G) . A$ monophonic domination set of $G$ is a sub set of $V(G)$ which is both monophonic and dominating set of G. The minimum cardinality of a monophonic domonation set is denoted by $\gamma_{\mathrm{m}_{\mathrm{e}}}(\mathrm{G})$. A detailed study of monophonic domination set is available in [3]. An edge monophonic set of $G$ is a subset $M \subset V(G)$ such that every edge of $G$ is contained in a monophonic path joining some vertices of $M$. The minimum cardinality among all the edge monophonic sets of $G$ is called edge monophonic number and is denoted by $m_{e}(G) A$ vertex $v$ is an universal vertex of a graph $G$ ifdeg $(v)=n-1$.

Edge monophonic set of a connected graph is studied in [2].

Remark 1.1:LetG be a connected graph of order $n \geq 3$. If $G$ contains exactly oneuniversal vertex, then $m_{e}(G)=n-1$ (see[2]).

\section{BASIC CONCEPTS AND DEFINITIONS}

Definition 2.1:A set of vertices D of a graph G is edge monophonic domination set(EMDset) if it is both edge monophonic set and a domination set of $G$. The minimum cardinality among all the EMD sets of $G$ is called edge monophonic domination number(EMD number) and is denoted by $\gamma_{\mathrm{m}_{\mathrm{e}}}(\mathrm{G})$.

Example 2.1 Consider the graph $\mathrm{G}$ given in figure 01. Here $\mathrm{M}=\left\{\mathrm{v}_{4}, \mathrm{v}_{7}, \mathrm{v}_{8}\right\}$ is an edge monophonic set . $\mathrm{N}=\left\{\mathrm{v}_{3}, \mathrm{v}_{5}, \mathrm{v}_{6}\right\}$ is adominating set and $\mathrm{D}=\left\{\mathrm{v}_{2}, \mathrm{v}_{4}, \mathrm{v}_{7}, \mathrm{v}_{8}\right\}$ is a minimum EMD set. Hence $\gamma_{\mathrm{m}_{\mathrm{e}}}(\mathrm{G})=4$. 


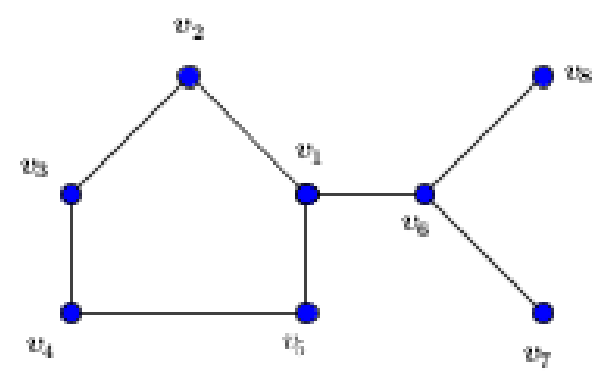

G Figure 01

Theorem 2.1:LetG be a connected graph. Then $\left.2 \leq \mathrm{m}_{\mathrm{e}}(\mathrm{G}) \leq \gamma_{\mathrm{m}_{\mathrm{e}}}(\mathrm{G})\right) \leq \mathrm{n}$.

Proof:Any edge monophonic set has atleast two vertices. There for $2 \leq m_{e}(G)$.Since every EMD set is an edge monophonic set $m_{e}(G) \leq \gamma_{m_{e}}(G)$. Also setof all vertices of $G$ induces the graph $G$, we have $\gamma_{m_{e}}(G) \leq n$.

Remark 2.1 :The bounds in theorem 2.1 are sharp. In figure $01,2<m_{\mathrm{e}}(\mathrm{G})<\gamma_{\mathrm{m}_{\mathrm{e}}}(\mathrm{G})<n$.

Theorem 2.2 :For any connected graph $\mathrm{G}$ of order $\mathrm{n}, 2 \leq \gamma_{\mathrm{m}}(\mathrm{G}) \leq \gamma_{\mathrm{m}_{\mathrm{e}}}(\mathrm{G}) \leq \mathrm{n}$

Proof : Since a monophonic domination set need atleast two vertices, $2 \leq \gamma_{\mathrm{m}}(\mathrm{G})$. Alsoevery EMD set is a monophonic domination set, $\gamma_{\mathrm{m}}(\mathrm{G}) \leq \gamma_{\mathrm{m}_{\mathrm{e}}}(\mathrm{G})$. Since the vertex set of $\mathrm{G}$ is both edge monophonic and domination set, $\gamma_{\mathrm{m}_{\mathrm{e}}}(\mathrm{G}) \leq \mathrm{n}$.

Remark 2.2:The bonds in theorem 2.2 are sharp. In figure $02, \gamma_{\mathrm{m}}(G)=3, \gamma_{\mathrm{m}_{\mathrm{e}}}(\mathrm{G})=4$ and $\mathrm{n}=6$.

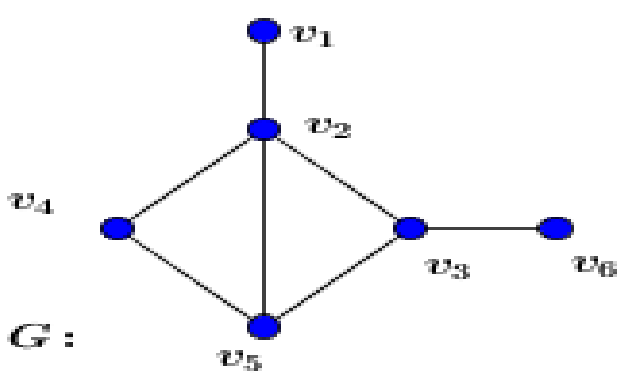

Figure 02:

Theorem2.3 :Each semi-extreme vertex of G belongs to every EMD set ofG.

Proof :LetD be an EMD set of G. Let $\mathrm{u}$ be a semi-extreme vertex ofG. Take $\mathrm{u} \notin \mathrm{D}$. Let $\mathrm{v}$ be a vertex of $<N(u)>$ such that $\operatorname{deg}_{<N(\mathrm{u})>}(\mathrm{v})=|\mathrm{N}(\mathrm{u})|-1$. Let $\mathrm{v}_{1}, \mathrm{v}_{2} \ldots \mathrm{v}_{\mathrm{k}}(\mathrm{k} \geq 2)$ be the neighbourhood of $\mathrm{v}$ in $\langle N(u)>$. Since $\mathrm{D}$ is also an edge monophonic set of $G$, the edge vu lies on the monophonic path $P: w, w_{1}, \ldots v_{i}, u, v, v_{j} \ldots t$, where $w, t \in D$. Since $u$ is a semi extreme vertex of $\mathrm{G}, \mathrm{v}$ andv $\mathrm{v}_{\mathrm{j}}$ are adjacent in $\mathrm{G}$ and so $\mathrm{P}$ is not a monophonicpath of $\mathrm{G}$. This contradicts our assumption.

Theorem 2.4 :For a semi extreme graph G of order $n, \gamma_{m_{e}}(G)=n$

Proof: Since each semi -extreme vertex belongs to every edge monophonicset and $V(G)$ is itself a domination set, the result follows.

Theorem 2.5 :Each extreme vertex of $\mathrm{G}$ belongs to every EMD set of G.

Proof: Since each extreme vertex of $\mathrm{G}$ belongs to every edge monophonic setof , the result follows.

Remark 2.3 :The set of all extreme vertices need not form an EMD set. Consider $\mathrm{P}_{\mathrm{n}}$ ofpath graph having more than four vertices.

Corollary 2.1 :Forthe complete graphK ${ }_{p}, \gamma_{m_{e}}(G)=p$. 
Theorem 2.6 :For cycle graph $C_{n}$ of $n$ verices, $\gamma_{m_{e}}\left(C_{n}\right)=2$, when $n \leq 6$ and it is equel to $[(n-r) \div 3]+1$ when $\mathrm{n}>6$, where $\mathrm{r}$ is the reminder when $\mathrm{n}$ isdivided by 3 .

Proof: Since G is a cycle, two non adjecent vertices in G defines an edgemonophonic set so that $m_{e}\left(C_{n}\right)=2$. Again each vertex dominates three vertices in a cycle, the result follows.

Theorem 2.7 :For the complete bipartite graph $K_{m, n}$

$\gamma_{m_{e}}(G)=\left\{\begin{array}{c}2, \quad \text { if } m=n=1 \\ n, \quad \text { if } n \geq 2, m=1 \\ \min \{m, n\}, \quad \text { if } m, n \geq 2\end{array}\right.$

Proof:(i) When $m=n=1: K_{m, n}=K_{2}$ complete graph of two vertices. Hence by corollary $2.1 \gamma_{m_{e}}(G)=2$.(ii) Here each $n$ verices are extreme vertices and belongs to every EMD set.(iii) Without loss of generality assume that $m \leq n$. Take $X=\left\{x_{1}, x_{2} \ldots x_{\mathrm{m}}\right\}, Y=\left\{y_{1}, y_{2}, \ldots y_{\mathrm{n}}\right\}$ be a partition of $G$. Consider $D=X$. Then $D$ is a minimumedge monophonic set (By Theorem 2.11 of [2]). Also the set $D$ dominateevery vertex in $G$ and is the minimum dominating set.Thus $D$ is a minimumEMD set. There for $\gamma_{m_{e}}(G)=|D|=m=\min \{(m, n\}$

Theorem 2.8 : Let $G$ be a connected graph, $u$ be a cut vertex of $G$ and let $D$ be an EMD set of $G$. Then every component of $G-u$ contains some verticesof $D$.

Proof: Let $u$ be a cut vertex of $G$ and $D$ be an EMD set of $G$. Let thereis some component, say $C_{1}$ of $G-u$ such that $C_{1}$ have no vertices of $D$.By theorem 2.4, $D$ contains all the extreme vertices of $G$ so that $C_{1}$ has noextreme vertex of $G$. Hence $C_{1}$ has an edge $a b$. Since $D$ is an EMD, $a b$ lieson some $v-w$ path $P: v, v_{1}, v_{2} \ldots u, \ldots a, b, \ldots u_{1} \ldots u \ldots w$ which is monophonic.Since $u$ is a cut vertex of $G$,every path traverse through $u$. Then $v-a$ and $b-w$ are sub paths of $P$ both contains $u$. There for $P$ is not a path which is acontradiction.

Theorem 2.9 : Let $T$ be a tree such that $N(x)$ belongs to end vertices forevery internal vertex $x \in T$.Then EMD number is equal to the number ofend vertices in $T$

Proof: Let $D$ be the set of all end vertices of $T$. Since each extreme vertexbelongs to EMD set of $T, D$ is the subset of every EMD set of $T$. That is $\gamma_{m_{e}}(T) \geq|D|$. The converse is trivial.

Theorem 2.10 :Let $G$ be a connected graph of order $n$.If there exist a uniquevertex $v \in V(G)$ such that $v$ is not a semiextreme vertex of $G, \gamma_{m_{e}}(G)=n-1$.

Proof : If $G$ is a connected graph having a unique non semi-extreme vertex $v$, then edge monophonic number of $G$, $m_{\mathrm{e}}(G)=n-1$ by theorem 2.19 of[2].Now every $n-1$ vertices of a graph is always a domination set, these $n-1$ vertices form a minimum EMD set.

Corollary 2.2 :Let $G$ be a connected graph of order $n \geq 3$.If $G$ contains exactly oneuniversal vertex, then $\gamma_{m_{e}}(G)=$ $n-1$.

Corollary 2.3 :For the wheel graph $W_{1, n-1}$ with $n \geq 4 ; \gamma_{m_{e}}\left(W_{1, n-1}\right)=n-1$.

Theorem 2.11 : Let $G$ be a connected graph of order $n \geq 2$, then $\gamma_{m_{e}}(G)=2$ if and only if there exist an edge monophonic set $D=\left\{x_{1}, x_{2}\right\}$ of $G$ such that $d_{\mathrm{m}}\left(x_{1}, x_{2}\right) \leq 3$.

Proof :Let $\gamma_{m_{e}}(G)=2$.Take $D=\left\{x_{1}, x_{2}\right\}$ as an EMD set.If $d_{\mathrm{m}}\left(x_{1}, x_{2}\right) \geq 4$, then the diametrical path contains atleast three internal vertices. Then $\gamma_{m_{e}}(G) \geq 3$ and is a contrediction. Thus $d_{\mathrm{m}}\left(x_{1}, x_{2}\right) \leq 3$. Conversly, let $d_{\mathrm{m}}\left(x_{1}, x_{2}\right) \leq 3$.If $D=$ $\left\{x_{1}, x_{2}\right\}$ is an edge monophonic set, then it is also adominatingset. Therefor $\gamma_{m_{e}}(G)=2$.

\section{REALIZATION RESULTS}

Theorem 3.1 :For any two integers $p, q \geq 2$, there exist a connected graph $G$ such that $\gamma(G)=p, m_{\mathrm{e}}(G)=q$ and $\gamma_{m_{e}}(G)=p+q$.

Proof : Consider $C_{5}$ with vertex set $\left\{c_{1}, c_{2}, c_{3}, c_{4}, c_{5}\right\}$. Let $A$ be the graphobtained by adding $q-1$ vertices $x_{1}, x_{2} \ldots x_{\mathrm{q}-1}$ with $C_{5}$ and join them at thevertex $c_{1}$. Let $G$ be the graph obtained from $A$ by adding a path of $3(p-2)+1$ vertices say $w_{0}, w_{1}, w_{2}, \ldots w_{3(\mathrm{p}-2)}$ where $w_{0}$ is adjecent with $c_{3}$ ( figure03). 


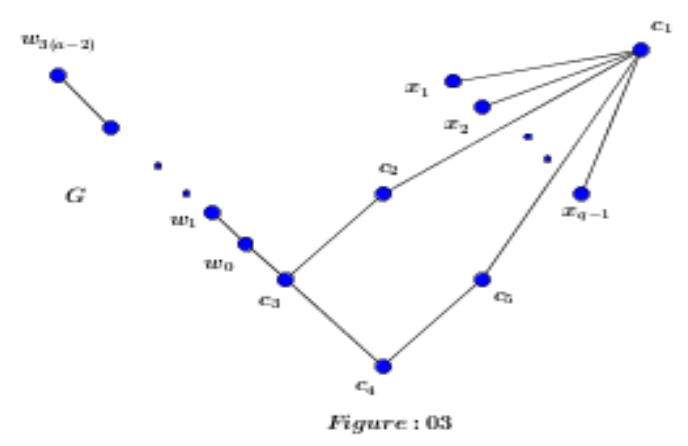

Let $E=\left\{c_{1}, c_{3}, w_{2}, w_{5} \ldots w_{3(\mathrm{p}-2)}\right\}$. Then $E$ is a minimum dominating set of $G$.Clearly $E$ contains $p$ vertices so that $\gamma(G)=$ $p$. Take $F=\left\{x_{1}, x_{2}, \ldots x_{\mathrm{q}-1}, w_{3(\mathrm{p}-2)}\right\}$. Then $F$ is a minimum edge monophonic set of $G$. Thus $m_{\mathrm{e}}(G)=q$. Now $D=\left\{x_{1}, x_{2} \ldots x_{\mathrm{q}}\right.$ $1, w_{2}, w_{5} \ldots w_{\left.3(\mathrm{p}-2), C_{1}, c_{3}\right\}}$ is a minimum EMD set sothat $\gamma_{m_{e}}(G)=p+q$.

Theorem 3.2:Forfor any two integers $p$ and $q$ such that $2 \leq p \leq q$ there exist a connected graph $G$ with $\gamma_{m}(G)=p$ and $\gamma_{m_{e}}(G)=q$.

Proof:Consider the following cases.

Case 1 : Let $p \geq 3, q \geq 4, q \neq p+1$.

Take $\mathrm{G}$ as the graph given in figure 04.Now $\mathrm{G}$ is obtained by adding two setof vertices $\left\{\mathrm{x}_{1}, \mathrm{x}_{2} \ldots \mathrm{x}_{\mathrm{p}-1}\right\}$ and $\left\{\mathrm{y}_{1}, \mathrm{y}_{2} \ldots \mathrm{y}_{\mathrm{q}-\mathrm{p}-1}\right\}$ with the path $P$ : $u, v, w$ in $G$ such a way that each $x_{i}$ join with $u$ and $v$ and each yjoin with $u, v, w$ butnot mutually. Let $D_{1}=$ $\left\{\mathrm{x}_{1}, \mathrm{x}_{2} \ldots \mathrm{x}_{\mathrm{p}-1}, \mathrm{w}\right\}$. Then $\mathrm{D}_{1}$ is a minimum monophonic domination set of $\mathrm{G}$. Therefore $\gamma_{\mathrm{m}}(\mathrm{G})=\mathrm{p}$. Since $\mathrm{v}$ is the unique universal vertex, by corollary2.2 $\gamma_{\mathrm{m}_{\mathrm{e}}}(\mathrm{G})=|\mathrm{G}|-1=(\mathrm{q}-\mathrm{p}-1+\mathrm{p}-1+3)-1=\mathrm{q}$.
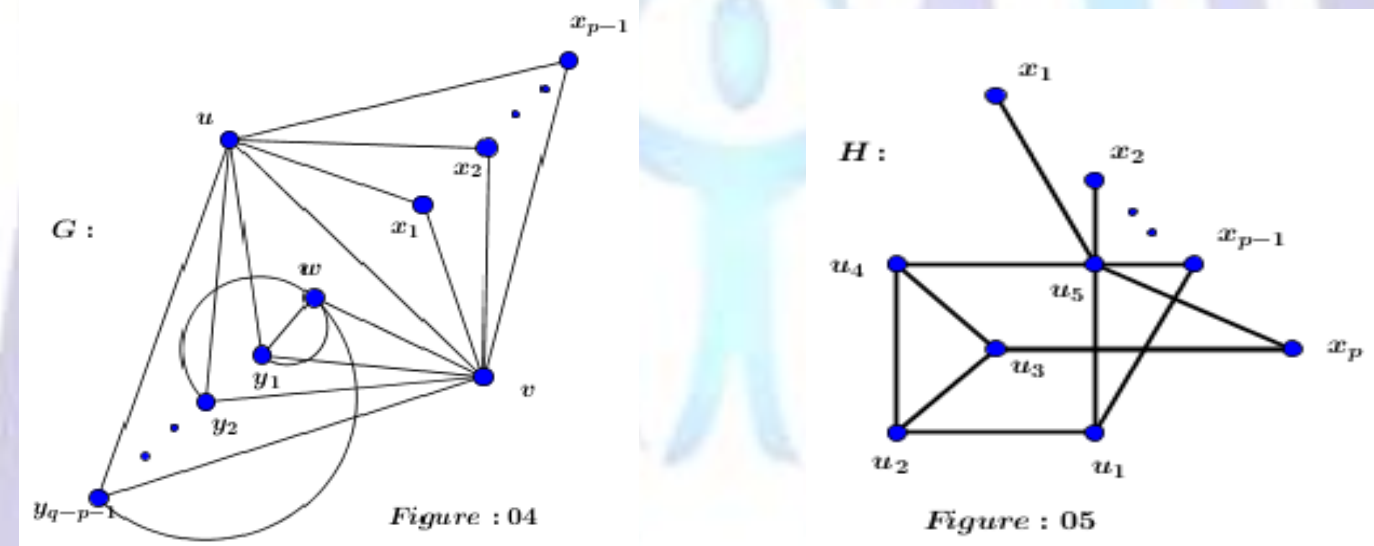

Case 2:p $\geq 3, q \geq 4, q=p+1$

Consider the following graph $\mathrm{H}$ ( figure 05 ). Take $\mathrm{A}=\left\{\mathrm{x}_{1}, \mathrm{x}_{2} \ldots \mathrm{x}_{\mathrm{p}-1}, \mathrm{v}_{3}\right\}$. It is a minimum monophonic domination setof $\mathrm{H}$. Therefor $\gamma_{\mathrm{m}_{\mathrm{e}}}(\mathrm{H})=\mathrm{p}$. Now $\mathrm{A}$ is not an EMD set since the edge $\mathrm{u}_{2} \mathrm{u}_{4}$ isnot lies in any edge monophonic path. But $\mathrm{A} \cup\left\{\mathrm{u}_{2}\right\}$ is an EMD set. Therefor $\gamma_{m_{e}}(H)=(p-1+1)+1=p+1=q$.

Case 3: Let $\mathrm{p}=2, \mathrm{q} \geq 4$.

Consider the graph $\mathrm{K}$ given in figure 06 .Kis obtained using the path $\mathrm{P}: \mathrm{u}, \mathrm{v}, \mathrm{w}$ of three vertices, by adding $\mathrm{q}-2$ new vertices $\mathrm{x}_{1}, \mathrm{x}_{2} \ldots \mathrm{x}_{\mathrm{q}-2}$ and join these vertices with $\mathrm{u}, \mathrm{v}, \mathrm{w}$. Here $\mathrm{v}$ is auniversal vertex. Therefore $\gamma_{\mathrm{m}_{\mathrm{e}}}(\mathrm{K})=|\mathrm{K}|=\mathrm{q}-2+3-$ $1=$ q. But $\mathrm{A}=\{\mathrm{u}, \mathrm{w}\}$ is a monophonicdomination set of $\mathrm{K}$. Therefore the edge monophonic domination number $\gamma_{\mathrm{m}_{\mathrm{e}}}(\mathrm{K})=2$.

Case 4: Let $\mathrm{p}=2, \mathrm{q}=3$.

Consider the graphL given in figure 07. Here $A=\left\{x_{2}, x_{5}\right\}$ is a monophonic domination set but not an EMD set.Therefor $\gamma_{m}(L)=p$. Take $D=\left\{x_{1}, x_{3}, x_{4}\right\}$. It is a minimum EMD set. Therefor $\gamma_{m_{e}}(L)=3$.

Case 5 : Let $\mathrm{p}=\mathrm{q}$.Take $\mathrm{T}$ as the bipartrite graph $\mathrm{K}_{1, \mathrm{p}}$. Then $\gamma_{\mathrm{m}}(\mathrm{L})=\gamma_{\mathrm{m}_{\mathrm{e}}}(\mathrm{L})=\mathrm{p}$. 


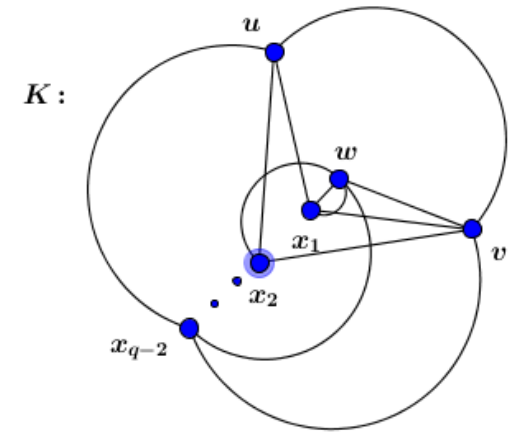

Figure : 06

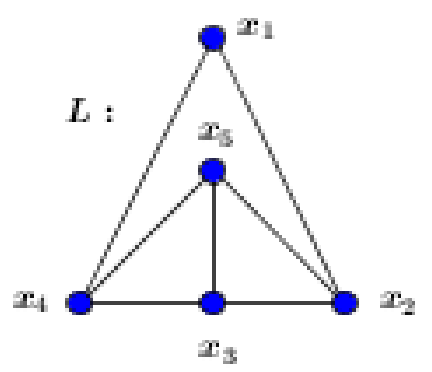

Figure 07

\section{CONCLUSION}

The results used in this article can extended to find properties of upper EMDset,forcing EMD set and EMD number of join of graphs, EMD number ofcomposition of graphs and EMD hull number of graphs and so on.

\section{REFERENCES}

[1] F.Buckley, and F.Harary. Distance in Graphs Addition Wesley. Red wood City(1990).

[2]J.Jhon and P.Arul Paul Sudhahar. On The Edge Monophonic Number ofaGraph.Filomat. Vol.26.6 pp 1081-1089(2012)

[3]J.JhonandP.Arul Paul Sudhahar. The Monophonic Domination Numberof a Graph.Proceedings of the International Conference on MathematicsandBuisnessManagement.(2012) pp 142-145.

[4] A.P Santhakumaran, P. Titus and R. Ganesamoorthy.OnThe Monophonic Number of a Graph Applied Math and Informatics. Vol 32,pp255-266 (2014)

\section{Authors' biography}
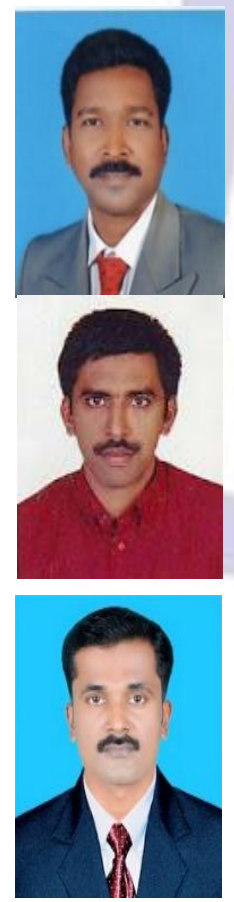

\section{Dr. P Arul Paul Sudhahar. P hD. M Sc. M Phil.}

Working as an Assistant Professor in the Department of Mathematics, Rani Anna Govt. College (W), Tirunelveli, TAMILNADU, INDIA and Research Supervisor under the Alagappa University, Karaikkudi, TAMILNADU, INDIA.

\section{Mr. M Mohammed Abdul Khayyoom M.}

Working as Higher Secondary School Teacher in the Govt. VHSS Omanoor, Malappuram-673 645, KERALA,INDIAand Research Scholar in the Alagappa University, Karaikkudi, TAMILNADU,INDIA under the Supervision of Dr. P Arul Paul Sudhahar.

\section{Mr. A Sadiquali.}

Working as an Assistant Professor \& Head in the Department of Mathematical Science, MEA Engineering College, Perinthalmanna-679325 KERALA, INDIA and Research Scholar in the Alagappa University, Karaikkudi, TAMILNADU, INDIA under the Supervision of Dr. P Arul Paul Sudhahar. 